\title{
BMJ Open Systematic review of the effects of iodised salt and iodine supplements on prenatal and postnatal growth: study protocol
}

\author{
Jessica Farebrother, ${ }^{1}$ Celeste E Naude, ${ }^{2}$ LiesI Nicol, ${ }^{2}$ Zhongna Sang, ${ }^{3}$ \\ Zhenyu Yang, ${ }^{4}$ Maria Andersson, ${ }^{1}$ Pieter L Jooste, ${ }^{5}$ Michael B Zimmermann ${ }^{1}$
}

To cite: Farebrother J, Naude CE, Nicol L, et al. Systematic review of the effects of iodised salt and iodine supplements on prenatal and postnatal growth: study protocol. $B M J$ Open 2015;5:e007238. doi:10.1136/bmjopen-2014007238

- Prepublication history and additional material is available. To view please visit the journal (http://dx.doi.org/ 10.1136/bmjopen-2014007238)

Received 18 November 2014 Revised 24 February 2015 Accepted 27 February 2015

CrossMark

For numbered affiliations see end of article.

\section{Correspondence to} Jessica Farebrother; jessica.farebrother@hest.ethz. ch

\section{ABSTRACT}

Introduction: Iodine is an essential micronutrient and component of the thyroid hormones. Sufficient ingestion of iodine is necessary for normal growth and development. If iodine requirements are not met, growth can be impaired. Salt iodisation and supplementation with iodine can prevent iodine deficiency disorders and stunted growth. No systematic review has yet collated the evidence linking iodine to growth. With an increased emphasis on stunting within the WHO Global Nutrition Targets for 2025, we propose a systematic review to address this question.

Methods and analysis: We will undertake a systematic review, and if appropriate, meta-analyses, evaluating the effects of iodised salt or iodine supplements on prenatal and postnatal somatic growth, until age 18. We will search a number of databases, including MEDLINE, EMBASE, Web of Science, CINAHL, PsychINFO, the Cochrane Library, including the CENTRAL register of Controlled Trials and also the WHO library and ICTRP (International Clinical Trials Registry Platform), which includes the Clinicaltrials.gov repository. We will also search Wanfang Data and the China Knowledge Resource Integrated Database. Included studies must have compared exposure to iodised salt, iodine supplements or iodised oil, to placebo, non-iodised salt or no intervention. Primary outcomes will be continuous and categorical markers of prenatal and postnatal somatic growth. Secondary outcomes will cover further measures of growth, including growth rates and indirect markers of growth such as insulinlike growth factor-1 (IGF-1).

Ethics and dissemination: The systematic review will be published in a peer-reviewed journal, and will be sent directly to the WHO, United Nations Children's Fund, International Council for the Control of lodine Deficiency Disorders and other stakeholders. The results generated from this systematic review will provide evidence to support future programme recommendations regarding iodine fortification or supplementation and child growth.

Trial registration number: PROSPERO CRD42014012940.

\section{Strengths and limitations of this study}

- This will be the first systematic review to investigate the effects of all forms of iodine supplementation or fortification on somatic growth, in the relevant population groups.

- A great deal of research on iodine nutrition has been conducted in specific provinces in China. To ensure that we are comprehensive and capture all relevant data, we will search Chinese bibliographic databases in addition to the large English (or other Latin alphabets) bibliographic databases such as MEDLINE and EMBASE.

- This systematic review addresses a high-priority problem (ie, the prevention and treatment of stunting) in the field of child and maternal health and nutrition. The results are potentially useful to inform iodine-related guidelines and recommendations made by key stakeholders involved in improving child and maternal health outcomes.

- This protocol has been written following the PRISMA-P guidelines recently developed by the PRISMA Group, ${ }^{12}$ and we will conduct this systematic review in line with the rigorous Cochrane methodology. Our international, expert review team brings both subject and methods expertise to the project. Given that much of the significant research contributions in the field of iodine nutrition are older, and that randomised trials are often not available to assess the effects of iodine-related fortification and supplementation interventions, we will include a broader range of non-randomised study designs to fully evaluate the current available evidence for our question. While non-randomised studies may provide valuable evidence on the effects of interventions, their inclusion introduces a greater risk of bias, specifically systematic selection bias, which can give rise to confounding threatening internal validity. Risk of bias and quality of evidence assessments (GRADE) will be carefully considered when interpreting data, reporting findings and formulating conclusions.

\section{INTRODUCTION}

Micronutrient deficiencies are important contributors to the global burden of disease and 
disability $^{3}$ One of the most widespread micronutrient deficiencies worldwide is that of iodine. The WHO estimates that approximately 285 million, or $37 \%$ of schoolage children and nearly two billion individuals worldwide have insufficient iodine intake. ${ }^{4}$

Iodine is required for normal physical growth during gestation and early life, and it is an essential component of the hormones produced by the thyroid gland. When dietary iodine requirements are not met, synthesis of the thyroid hormones is impaired. The resulting hypothyroidism induced by iodine deficiency during pregnancy, infancy and childhood can impair growth and development..$^{5-8}$ The reduced activity of thyroid hormones resulting from iodine deficiency, reduces the effects of growth hormone (either through effects on pituitary secretion or at its receptor) and as well as circulating concentrations of insulin-like growth factor (IGF-1) and its binding proteins. ${ }^{9}{ }^{10}$ Iodine deficiency can result in a number of developmental and functional abnormalities, the spectrum of which is referred to as the iodine deficiency disorders (IDD). ${ }^{69}$

Salt iodisation has been recommended as a safe and cost-effective primary strategy to ensure sufficient iodine intake by all individuals, including pregnant women and children. ${ }^{6}$ In situations where the coverage of iodised salt is incomplete, daily iodine supplementation is recommended to pregnant women, lactating women and infants. ${ }^{6}$ Periodic doses of iodised oil may also be an effective intervention in vulnerable groups until iodised salt can be implemented. ${ }^{6}$ Iodine may also be provided through fortification of certain foodstuffs, including milk and bread and water.

There is an increased emphasis on stunting on the global nutrition agenda, and stunting will likely be a leading post-Millennium Development Goal. ${ }^{11}{ }^{12}$ Consequently, the roles of nutrition interventions, including micronutrient strategies for stunting have recently received attention from global decision-makers, such as the WHO. There is a need to examine this evidence base $^{13}$ in line with recent efforts to promote evidenceinformed guideline development for nutrition. ${ }^{14}$ Within this context, the evidence on the role of iodine in prenatal and postnatal growth has not received much focus to date. The prevention and correction of iodine deficiency may be an important contributor to reducing stunting, as part of a multiple intervention package for improving child health.

To avoid duplication and investigate the need for a new systematic review on this question, we first conducted at scoping review, ${ }^{15}$ and identified 10 existing systematic reviews, ${ }^{16-25}$ that investigate the effect of iodine supplementation on cognitive development, thyroid function, iodine deficiency disorders and certain growth outcomes in children and adults. None of the identified systematic reviews investigated the effects of all forms of iodine fortification/ supplementation in all of the relevant population groups (ie, women of childbearing age, pregnant and lactating women and children of all ages) on all of the relevant growth and growth-related outcomes.
A previous Cochrane systematic review published in $2002,{ }^{23}$ concluded that iodised salt was an effective means of improving population iodine status and found no adverse effects of iodine supplementation or fortification. However, there was insufficient evidence to make conclusions regarding outcomes, such as childhood growth or cognition. Since the review in 2002, many additional studies have been published which may provide information on these developmental outcomes. A recent update of the above Cochrane Review, ${ }^{26}$ included studies investigating the effect of iodised salt only, in children and adults on outcomes including all-cause mortality, goitre, cognitive function and cretinism. The update did not examine the effects of all forms of iodine supplementation on foetal or infant growth, either prenatal or postnatal.

Iodine deficiency has been and remains a major problem in certain regions of China, such as the Gansu, Yunnan and Sichuan provinces. There has been much research aimed at improving iodine nutrition in these areas and elsewhere in China, which represents an important component of iodine literature worldwide. Iodine intervention studies undertaken in China may not have been captured in previous systematic reviews published in English or other Latin alphabets, or in reviews where searches were not undertaken specifically in Chinese databases: our scoping review, ${ }^{15}$ found that Chinese databases had been specifically searched in only 2 of the 10 systematic reviews identified.

Data from both observational and experimental studies have described the relationship between iodine nutrition and somatic growth, ${ }^{70}$ 27-29 however this literature has never been systematically evaluated with respect to this specific question. A systematic review of studies investigating the effects of all forms of iodine supplementation and fortification on prenatal and postnatal somatic growth in relevant population groups is clearly required to interpret the existing body of evidence addressing this question, and possibly identify gaps for future research. The objective of this systematic review is therefore to assess the effects of iodised salt, iodine supplements and iodised oil compared to placebo or no intervention on somatic growth of the fetus, infant and child, and define the magnitude of the effect on growth after iodine repletion.

\section{METHODS AND ANALYSIS}

In line with Cochrane recommendations, our review team includes both subject experts and researchers with expertise in systematic review methodology. ${ }^{30}$ One core review team (CEN, LN and JF) will be responsible for the review of literature in English and other Latin alphabet texts. Two Chinese reviewers also proficient in English (ZS and ZY) will comprise another core review team, being specifically responsible for all literature in Chinese. A common, standardised work plan has been established to streamline all processes within both groups, which will be led by one reviewer (JF). An advisory group consisting of the three senior authors and a 
senior methods expert will guide the process. A consultant biostatistician has reviewed the protocol and relevant additional statistical expertise will be sought, as needed. The review will be performed following Cochrane methodology. ${ }^{30} 31$ The PRISMA (Preferred Reporting Items for Systematic Reviews and Meta-Analyses) ${ }^{32}$ statement will be used for the reporting of the review.

We formulated the following research question: "What are the effects of iodised salt, iodised oil or iodine supplements compared to placebo or no iodine intervention on prenatal and postnatal somatic growth of the foetus, infant, child and adolescent?"

Our systematic review is registered with PROSPERO, registration number CRD42014012940, available at http://www.crd.york.ac.uk/PROSPERO.

\section{Criteria for considering studies for inclusion \\ Types of studies}

We will include randomised controlled trials (RCTs), and non-randomised controlled trials (non-RCTs), controlled before-after (CBA) studies, interrupted time-series studies (ITS) and interrupted time series studies with repeated measures (RMS) ${ }^{33}$ All studies must have compared a group of individuals with exposure to iodised salt, iodine supplements or iodised oil to a group receiving a placebo, non-iodised salt or no intervention. In the case of ITS and RMS studies, observations at multiple time points before and after an intervention must be included in the study design. ITS and RMS that do not have a clearly defined point in time when the intervention occurred and at least three data points before and three after the intervention will be excluded, as multiple data points are required to detect whether the intervention has had an effect significantly greater than any underlying trend over time. Trials can be of a parallel or crossover design. For crossover studies the data from the first period must be available.

\section{Types of participants}

For prenatal growth, the populations will be pregnant women or women of childbearing age, and their offspring. For postnatal growth, the participants will be lactating women and their breastfeeding infant considered as one unit, in addition to children of any age (0-18 years) and of either gender. Study populations could be within the general population or part of specific populations such as refugee populations. Studies looking specifically at the link between growth and populations affected by congenital hypothyroidism and goitre will be excluded. Similarly, studies looking at non-iodine-related growth will be excluded. Furthermore, studies reporting on pregnant women who smoke will be excluded, since a correct assessment of the effects of iodine on growth in this population cannot be adequately assessed.

\section{Types of interventions and comparisons}

This review will consider exposure to iodised salt, iodine supplementation or iodised oil in any form, dose and regimen (including multinutrient interventions) compared to a placebo, non-iodised salt or no intervention. The populations being compared should have a similar baseline iodine status, for example, being from the same geographical area with a known iodine status, and they must be comparable. Comparisons featuring populations that are from areas with a different baseline iodine status, that is, from an area of known deficiency in comparison to a region of established excess, will be excluded. Multinutrient interventions where the effect of iodine alone cannot be assessed will be excluded.

\section{Types of outcomes}

We will include studies that adhere to the descriptions above and that report any of the following outcomes:

Primary outcomes:

- Prenatal somatic growth (measured, eg, using birth weight and birth weight indices (such as intrauterine growth restriction, small-for-gestational age low birthweight, ponderal index), head circumference), in addition to in utero ultrasound measurements such as biparietal diameter) and measurements made during pregnancy, for example symphysis-fundal height.

- Postnatal somatic growth (measured, eg, using anthropometry (head circumference, weight-for-age, height or length-for-age, weight-for-height, BMI-for-age, midupper arm circumference, child growth rate)).

Secondary outcomes:

- Cretinism;

- Pregnancy weight gain;

- Indirect markers of somatic growth (insulin-like growth factor (IGF)-1; insulin-like growth factor binding protein (IGFBP)-3, Growth Hormone);

- Postnatal bone maturation;

- Malnutrition disorders (kwashiorkor, marasmus).

\section{Search methods for identification of studies Electronic searches}

A comprehensive search strategy was compiled in consultation with an expert librarian skilled in systematic review searching (see appendix). Using no date or language restrictions, the librarian will use the search strategy to search the following databases:

- MEDLINE

- EMBASE

- WEB OF SCIENCE (Social Sciences Citation index/ Science Citation Index)

- Cochrane Library, including CENTRAL register of Controlled Trials

- CINAHL (Cumulative Index to Nursing and Allied Health Literature)

- WHO International Clinical Trials Registry Platform (ICTRP; which includes http://www.clinicaltrials.gov) for on-going studies

- WHO library

- PsycINFO 
The search terms will be translated into Chinese, and we will search the following databases for records in Chinese, with no date restrictions:

- China Knowledge Resource Integrated Database (http://www.cnki.net), which includes the databases:

- China Academic Journals Full-text Database

- China Doctoral Dissertations Full-text Database

- China Masters Theses Full-text Database

- China proceedings of Conferences Full-text Database

- International Proceedings of Conferences Full-text Database

- Wanfang Data (http://www.wanfangdata.com), which includes the databases:

- China Online Journals

- Dissertations of China.

\section{Searching other resources}

For assistance in identifying ongoing or unpublished studies, we will contact the Sprinkles Global Health Initiative, the In-Home Fortification Technical Advisory Group, the nutrition section of the United Nations Children's Fund (UNICEF), the World Food Programme (WFP), the Micronutrient Initiative (MI), the Global Alliance for Improved Nutrition (GAIN), Helen Keller International (HKI), Sight and Life Foundation, the Department of Nutrition for Health and Development, WHO, the US Centers for Disease Control and Prevention (CDC) and the International Council for the Control of Iodine Deficiency Disorders (ICCIDD) Global Network.

We will contact experts in the field of iodine nutrition concerning studies published in non-peer-reviewed literature for example, book chapters. We will search the reference lists of the systematic reviews included in our scoping review, ${ }^{15}$ and any other existing systematic reviews identified during screening for eligible studies. Additionally, we will search the Chinese literature within the repository of full-text studies yielded from searches for a previous similar systematic review. ${ }^{26}$

\section{Data collection and analysis}

Selection of studies

Two reviewers from each core review team will independently screen the titles and abstracts of all records yielded by the respective searches. The reviewers from both teams will apply the prespecified criteria to identify potentially eligible studies for inclusion.

Full-texts articles of all potentially eligible studies identified by either of the reviewers will be retrieved for closer inspection. Full texts that are excluded after closer inspection will be listed in the table 'Characteristics of Excluded Studies', together with the reasons for their exclusion. The Covidence software platform, ${ }^{34}$ will be used to facilitate independent screening and selection, and to identify screening and inclusion discrepancies. Any discrepancies not resolved within each core review team, will be resolved through discussion with the other core review team and the advisory group.

\section{Data extraction and management}

Two reviewers from each core review team will independently extract relevant data from all eligible studies using a standardised data extraction form based on a good practice data extraction form for systematic reviews. ${ }^{35}$ Discrepancies regarding extracted data will be resolved as before, by discussion with the other core review team and advisory group, as necessary. All data not presented in English will be translated.

The following will be extracted from each included study:

- Administrative details: Trial identification number; author(s); published or unpublished; year of publication; number of studies included in paper; year in which study was conducted; details of other relevant papers cited;

- Details of the study: study design (eg, RCT, CBA, ITSS); type, duration and completeness of follow-up; country and location of study (eg, higher-income vs lowerincome country); representativity (eg, local, regional or national level), informed consent and ethics approval;

- Details of participants: age, sex, sample size, relevant baseline characteristics including iodine status (UIC) and thyroid hormone concentrations (Tg, TSH, T4, T3); prevalence of hypothyroidism, hypothyroxinemia, hyperthyroidism;

- Details of intervention and control group: type (iodate/ iodide) and level of iodine fortification, type of iodine supplements, dosage of supplement, form and formulation of supplement, additional cointerventions (such as fortification with micronutrients); treatment received by the control group.

- Details of outcomes: all prespecified outcomes and any additional outcomes reported in the study, adverse events and toxicity.

- Details of quality assessment: quality assessment of the study based on the Risk of Bias tool.

- Details of data analysis: numbers and reported statistics for each reported outcome.

Where trials are reported in more than one reference, relevant data from all trial reports will be extracted as comprehensively as possible, and the most recent effect sizes used in analyses, as appropriate. The Covidence software platform, ${ }^{34}$ will be used to compare double data extraction online. Where relevant, data from Covidence will be exported into Review Manager 5.1 for analysis. ${ }^{36}$

\section{Assessment of risk of bias in included studies}

Two reviewers from each core review team will independently assess the risk of bias of each included study using the EPOC Risk of Bias criteria for RCTs, non-RCTs, CBA studies and ITS. ${ }^{37}$ The Covidence software platform, ${ }^{34}$ will be used to compare methodological quality assessment and discrepancies resolved through discussion and consultation with the advisory group.

Measures of treatment effect

For dichotomous outcomes, where possible, the risk ratio (RR) from statistical analyses, adjusting for baseline 
differences (such as Poisson regressions or logistic regressions), or the ratio of RRs (ie, the RR postintervention/ RR preintervention) will be calculated using Review Manager 5.1. ${ }^{36}{ }^{38}$ For continuous variables, if possible, the weighted mean difference (WMD) with $95 \%$ CIs, the absolute change from a statistical analysis adjusting for baseline differences (such as regression models, mixed models or hierarchical models) or the relative change adjusted for baseline differences in the outcome measures (ie, the absolute postintervention difference between the intervention and control groups-the absolute preintervention difference between the intervention and control groups)/the postintervention level in the control group) will be calculated by meta-analysis. If necessary, standardisation of continuous outcomes across studies will be performed prior to meta-analysis (eg, as standardised mean differences). ${ }^{38}$ Statistical expertise will be sought for conducting meta-analysis of standardised outcomes, and for all other complex analyses, if required.

For ITS and RMS we will use either a regression analysis with time trends before and after the intervention, adjusting for autocorrelation and any periodic changes, or the ARIMA (autoregressive integrated moving average) analysis, which is used in time series analyses to understand or predict future trends. Where possible, the results for the outcomes will be presented as changes along two dimensions, namely change in level and change in slope. For ITS and RMS that do not define a transition phase, a transition phase will be defined and transition phase data will be excluded. ${ }^{38}$ If papers with ITS design do not provide an appropriate analysis or reporting of results, but present the data points in a table or in a graph that can be scanned, wherever possible, we will reanalyse the data using methods described in Ramsay et al. ${ }^{39}$

In RMS, the data are repeated outcome measures from many individuals. If a study does not report appropriate results, we will not reanalyse the data from the summary graphs, because no estimate of within-patient variability can be obtained from the summary graphs and any reanalysis would underestimate or overestimate the SE of the effect sizes. Therefore, we will only present the results reported in the original papers. ${ }^{38}$

\section{Unit of analysis errors}

For clustered designs (such as cluster randomised trials) the reported results in included studies will often be on another level than the level of allocation. If this is the case, an analysis adjusting for clustering will be performed in order to avoid unit-of-analyses errors. When extracted results are not based on analyses adjusted for clustering, a re-analysis of the results will be conducted. If there is a unit of analysis error in the reported analysis for a study and there is insufficient information to re-analyse the results, the study authors will be contacted to obtain necessary data. If these data are not available, we will not report CIs or $p$ values for which there is a unit of analysis error. ${ }^{38}$

\section{Dealing with missing data}

Where data is missing or unclear, we will contact study authors wherever possible. When percentages were provided without denominators we will back-calculate to determine denominators. If only SEs or 95\% CIs are reported for means and no SDs, the SDs will be calculated as follows: $\mathrm{SD}=\mathrm{SEM} \times \mathrm{Square}$ root of sample size. If unable to obtain missing data, we will report the results that are available, provided they are not likely to be misleading (eg, if there is a unit of analysis error).$^{38}$

\section{Assessment of heterogeneity}

Studies will first be assessed for clinical heterogeneity by examining variability in the participants, interventions and outcomes. Where it is clinically meaningful to combine studies, we will conduct a meta-analysis using randomeffects model as we anticipate heterogeneity. We will further assess statistical heterogeneity in the meta-analysis study results using the $\chi^{2}$ test for heterogeneity (significance level $\mathrm{p}<0.1)$. Statistical heterogeneity will also be quantified with the $\mathrm{I}^{2}$ test, using the following guidelines for the interpretation of the $\mathrm{I}^{2}$ values: ${ }^{40} 0-40 \%$ : might not be important; 30-60\%: may represent moderate heterogeneity; 50-90\%: may represent substantial heterogeneity and $75-100 \%$ : considerable heterogeneity.

\section{Assessment of reporting biases}

Provided there are a sufficient number of studies we will use a funnel plot to assess the risk of publication bias.

\section{Data synthesis and investigation of heterogeneity}

Where studies are sufficiently homogenous the results of each of the prespecified outcomes reported in the studies will be combined using random-effects meta-analysis.

When data allow, the intervention effects across the following subgroups (explanatory factors) will be compared to explore heterogeneity:

- Iodised salt versus iodine supplements (eg, iodised oil)

- Age: prenatal (birth weight); infant (0-24 months); preschool child (2-5 years), school age child (6-12 years) and adolescent (13-18 years) .

These subgroups are selected following conventional classifications based on growth periods. Across all modes of iodine intake being investigated, with the exception of iodised oil, we expect a positive influence of iodine on growth equally across all age groups, thereby considering the bioavailability of iodine from salt, supplement, milk, bread or water to be equivalent. For iodised oil, the age subgroups become particularly important, as we expect the positive effect of iodine on growth to increase with age. This is due to the fact that individuals in older age groups will be more developed physically, including having a higher proportion of adipose tissue. Since iodised oil is stored in adipocytes, ${ }^{41}$ it follows that a greater proportion of a given iodised oil dose will be absorbed, thereby providing a more important store of iodine for a more prolonged release. 
If there are sufficient numbers of comparisons for similar outcomes across studies, we will use graphical displays (bubble and whisker plots) to visually explore heterogeneity of the results across studies. If appropriate, the visual analyses will be supplemented with multivariate statistical analyses (meta-regression), to examine how the size of observed effects is related to the specified subgroups (explanatory factors). ${ }^{38}$ Several methods for performing meta-regression exist and statistical expertise will be sought for selecting the most appropriate method and software.

\section{Sensitivity analysis}

To determine how robust and consistent the results are, sensitivity analyses will be conducted based on study design (RCT vs other) or risk of bias in study (high, medium, low risk of bias). If a number of eligible trials investigate the effect of iodine in combination with multinutrient interventions (fortification and supplementation) compared with multinutrient interventions alone, then a sensitivity analysis will be performed to assess the combined effect of iodine and multinutrient interventions versus iodine supplementation or fortification alone. In the event of missing data, we will perform sensitivity analyses for missing data by imputing a plausible range of assumptions. The potential implications of missing information will be discussed. In addition, any methodological decisions (eg, choice of intra cluster coefficient (ICC) for re-analysis of cluster randomised trials or inclusion/exclusion of studies from analyses based on prespecified criteria such dropout rates $>20 \%$ ) taken in the course of preparing the review will be checked for stability of results in a sensitivity analysis. ${ }^{38}$

\section{Quality of evidence using GRADE}

Quality of evidence will be assessed using Grading of Recommendations, Assessment, Development and Evaluation (GRADE), via the GRADEpro browser-based platform (http://www.gradepro.org last accessed 12 November 2014) ${ }^{42}$ will be used to create Summary of Findings tables for each comparison using the prepecified outcomes. In determining the level of evidence for each outcome, both the efficacy results and the assessment of the risk of bias will be integrated into a final assessment of the level of evidence and details of the decision will be provided.

\section{Current study status}

At time of submission, we are in the process of screening full texts for the review, and expect to start data extraction in April 2015. Study details will be continuously updated on PROSPERO, and any amendments made will be fully published there with the date of amendment and full rationale.

\section{DISCUSSION}

Our systematic review will aim to provide a comprehensive, systematic assessment of existing evidence on the effects of iodine, in the form of salt or supplement, on somatic growth outcomes, both prenatal and postnatal to the age of 18 years. It will also provide an evaluation of the quality of this evidence using GRADE. ${ }^{42}$ The results will be interpreted and presented using Summary of Findings tables that reflect both the magnitude of effect and confidence in that effect. Consequently, these tables provide concise summaries of the key information needed in the decision-making process and, in the context of guideline development, provide summaries of the key information underlying a recommendation. ${ }^{43}$ Thus, it is hoped that this review will address an identified gap in the evidence and support future recommendations and programme guidance regarding iodine fortification and supplementation, and child growth and health. We envision that the results will be particularly useful to the WHO and other international organisations working towards achieving the 2025 WHO Global Nutrition Targets.

\section{Author affiliations}

${ }^{1}$ Human Nutrition Laboratory, Institute of Food, Nutrition, and Health, ETH Zurich, Zurich, Switzerland

${ }^{2}$ Centre for Evidence-Based Health Care, Faculty of Medicine and Health Sciences, Stellenbosch University, Stellenbosch, South Africa

${ }^{3}$ Department of Nutrition and Food Hygiene, School of Public Health, Tianjin Medical University, Tianjin, China

${ }^{4}$ Key Laboratory of Trace Element Nutrition of the Ministry of Health, National Institute of Nutrition and Food Safety, Chinese Centre for Disease Control and Prevention, Beijing, China

${ }^{5}$ Faculty of Health Sciences, Centre of Excellence for Nutrition, North-West University, Potchefstroom, South Africa

Acknowledgements The authors would like to express their appreciation to Professor Taryn Young, senior methods expert, Ms Tonya Esterhuizen, biostatistician and Dr Vittoria Lutje, expert librarian, for their contribution to this protocol.

Contributors JF is corresponding author and guarantor of this protocol. JF, CEN, LN and MZ were involved in the formulation of the research question and developing the protocol, with inputs from PJ and MA and ZS and ZY as needed. JF, CEN and LN drafted the protocol manuscript. MZ, MA and PJ reviewed the manuscript, contributed to revisions and approved the final draft.

Funding This work is supported in equal parts by The Foundation for Growth Science, Japanand the ETH Global, Switzerland.

Competing interests None declared.

Provenance and peer review Not commissioned; externally peer reviewed.

Open Access This is an Open Access article distributed in accordance with the Creative Commons Attribution Non Commercial (CC BY-NC 4.0) license, which permits others to distribute, remix, adapt, build upon this work noncommercially, and license their derivative works on different terms, provided the original work is properly cited and the use is non-commercial. See: http:// creativecommons.org/licenses/by-nc/4.0/

\section{REFERENCES}

1. Moher D, Shamseer L, Clarke M, et al. Preferred reporting items for systematic review and meta-analysis protocols (PRISMA-P) 2015 statement. Syst Rev 2015;4:1.

2. Shamseer L, Moher D, Clarke M, et al. Preferred reporting items for systematic review and meta-analysis protocols (PRISMA-P) 2015: elaboration and explanation. BMJ 2015;349:g7647. 
3. WHO. Global health risks: mortality and burden of disease attributable to selected major risks. Geneva, Switzerland: World Health Organization, 2009.

4. Andersson M, Karumbunathan V, Zimmermann MB. Global iodine status in 2011 and trends over the past decade. J Nutr 2012;142:744-50.

5. Zimmermann MB. The effects of iodine deficiency in pregnancy and infancy. Paediatr Perinat Epidemiol 2012;26(Suppl 1):108-17.

6. World Health Organization, United Nations Children's Fund, International Council for the Control of lodine Deficiency Disorders. Assessment of iodine deficiency disorders and monitoring their elimination.A guide for programme managers. 3rd edn. Geneva, Switzerland: World Health Organization, 2007.

7. Alvarez-Pedrerol M, Guxens M, Mendez M, et al. lodine levels and thyroid hormones in healthy pregnant women and birth weight of their offspring. Eur J Endocrinol 2009;160:423-9.

8. Chaouki ML, Benmiloud M. Prevention of iodine deficiency disorders by oral administration of lipiodol during pregnancy. Eur $J$ Endocrinol 1994;130:547-51.

9. Zimmermann MB, Jooste PL, Pandav CS. lodine-deficiency disorders. Lancet 2008;372:1251-62.

10. Zimmermann MB, Jooste PL, Mabapa NS, et al. Treatment of iodine deficiency in school-age children increases insulin-like growth factor (IGF)-I and IGF binding protein-3 concentrations and improves somatic growth. J Clin Endocrinol Metab 2007;92:437-42.

11. Ruel MT, Alderman $\mathrm{H}$. Nutrition-sensitive interventions and programmes: how can they help to accelerate progress in improving maternal and child nutrition? Lancet 2013;382:536-51.

12. Stevens GA, Finucane MM, Paciorek CJ, et al. Trends in mild, moderate, and severe stunting and underweight, and progress towards MDG 1 in 141 developing countries: a systematic analysis of population representative data. Lancet 2012;380:824-34.

13. WHO. Global Nutrition Policy Review: what does it take to scale up nutrition action? Geneva, Switzerland: World Health Organization, 2013.

14. WHO. Nutrition Guidance Expert Advisory Group (NUGAG) Micronutrients subgroup-terms of reference. Geneva, Switzerland: World Health Orgaization, 2010.

15. Farebrother J, Naude CE, Nicol L, et al. lodine for prenatal and postnatal somatic growth: a rapid scoping of existing systematic reviews (forthcoming). 2014.

16. Angermayr L, Clar C. lodine supplementation for preventing lodine Deficiency Disorders in children. Cochrane Library 2004;(2): CD003819.

17. Best C, Neufingerl N, Del Rosso JM, et al. Can multi-micronutrient food fortification improve the micronutrient status, growth, health, and cognition of schoolchildren? A systematic review. Nutrition reviews 2011:69:186-204

18. Bougma K, Aboud FE, Harding KB, et al. lodine and mental development of children 5 years old and under: a systematic review and meta-analysis. Nutrients 2013;5:1384-416.

19. Gunnarsdottir I, Dahl L. lodine intake in human nutrition: a systematic literature review. Food Nutr Res 2012;56:19731. http:// dx.doi.org/10.3402/fnr.v56i0.19731

20. Ibrahim M, Sinn J, McGuire W. lodine supplementation for the prevention of mortality and adverse neurodevelopmental outcomes in preterm infants. Cochrane Database Syst Rev 2006;(2):CD005253.

21. Khor G, Misra S. Micronutrient interventions on cognitive performance of children aged 5-15 yrs in developing countries. Asia Pac J Clin Nutr 2012;21:476-86.

22. Taylor PN, Okosieme OE, Dayan CM, et al. Therapy of endocrine disease: Impact of iodine supplementation in mild-to-moderate iodine deficiency: systematic review and meta-analysis. Eur J Endocrinol 2014;170:R1-15.
23. Wu T, Liu GJ, Li P, et al. lodised salt for preventing iodine deficiency disorders. Cochrane Library 2002;(3):CD003204.

24. Zhou SJ, Anderson AJ, Gibson RA, et al. Effect of iodine supplementation in pregnancy on child development and other clinical outcomes: a systematic review of randomized controlled trials. Am J Clin Nutr 2013;98:1241-54.

25. Ristic-Medic D, Dullemeijer C, Tepsic J, et al. Systematic review using meta-analyses to estimate dose-response relationships between iodine intake and biomarkers of iodine status in different population groups. Nutr Rev 2014;72:143-61.

26. Aburto N, Abudou M, Candeias V, et al. Effect and safety of salt iodisation to prevent iodine deficiency disorders: a systematic review with meta-analyses. Geneva, Switzerland: WHO eLibrary of Evidence for Nutrition Actions (eLENA) World Health Organization, 2014.

27. Semba R, de Pee S, Hess S, et al. Child malnutrition and mortality among families not using adequately iodised salt in Indonesia. Am J Clin Nutr 2008;87:438-44.

28. Mohammed H, Marquis GS, Aboud FE, et al. Nutritional status and mental development of children under 5 years of age in Amhara Region of Ethiopia. FASEB 2013;27:845.1.

29. Rydbeck F, Rahman A, Grander M, et al. Maternal urinary iodine concentration up to $1.0 \mathrm{mg} / \mathrm{L}$ is positively associated with birth weight, length, and head circumference of male offspring. J Nutr 2014;144:1438-44.

30. Higgins JPT, Green S, eds. Cochrane handbook for systematic reviews of interventions Version 5.1.0 [updated Mar 2011]. The Cochrane Collaboration, 2011. http://www.cochrane-handbook.org.

31. Effective Practice and Organisation of Care (EPOC). 2014. http:// epocoslo.cochrane.org/epoc-specific-resources-review-authors

32. Moher D, Liberati A, Tetzlaff J, et al. Preferred reporting items for systematic reviews and meta-analyses: the PRISMA statement. PLoS Med 2009;6:e1000097.

33. Effective Practice and Organisation of Care (EPOC). What study designs should be included in an EPOC review and what should they be called? 2014 (cited 31 Oct 2014). http://epocoslo.cochrane. org/epoc-specific-resources-review-authors

34. The Alfred Hospital MU, National ICT Australia, University of London. Covidence software platform. Melbourne: Alfred Health, 2013.

35. Effective Practice and Organisation of Care (EPOC). Good practice data extraction form. 2014 (cited 31 Oct 2014). http://epocoslo. cochrane.org/epoc-specific-resources-review-authors

36. Review manager (RevMan) [Computer program]. Version 5.3. Copenhagen: The Nordic Cochrane Centre, The Cochrane Collaboration, 2014.

37. Effective Practice and Organisation of Care (EPOC). Suggested risk of bias criteria for EPOC reviews. 2014 (cited 31 Oct 2014). http:// epocoslo.cochrane.org/epoc-specific-resources-review-authors

38. Effective Practice and Organisation of Care (EPOC). Analysis in EPOC reviews. 2014. (cited 31 Oct 2014). http://epocoslo.cochrane. org/epoc-specific-resources-review-authors

39. Ramsay CR, Matowe L, Grilli R, et al. Interrupted time series designs in health technology assessment-lessons from two systematic reviews. Int J Technol Assess Health Care 2004;19:613-23.

40. Higgins JP, Thompson SG. Quantifying heterogeneity in a meta-analysis. Stat Med 2002;21:1539-58.

41. Wolff J. Physiology and pharmacology of iodized oil in goiter prophylaxis. Medicine 2001;80:20-36.

42. GRADEpro. GRADEpro. [Computer program on http://www. gradepro.org]. Version 12, November 2014. McMaster University, 2014.

43. Guyatt G, Oxman AD, Akl EA, et al. GRADE guidelines: 1. Introduction-GRADE evidence profiles and summary of findings tables. J Clin Epidemiol 2011;64:383-94. 